https://doi.org/10.48009/2_iis_2010_176-183

\title{
HEALTHCARE INFORMATION SYSTEMS: A PEDAGOGICAL ANALYSIS OF CURRENT DEGREE PROGRAMS
}

\author{
Someswar Kesh, University of Central Missouri kesh@ucmo.edu \\ Sam Ramanujan, University of Central Missouri ramanujan@ucmo.edu
}

\begin{abstract}
This research provides a pedagogical analysis of the current healthcare information systems degree programs from various U.S. universities and classifies the coursework offered in these universities into common themes. It is expected that universities planning to offer degree programs in healthcare information systems can use these themes as the fundamental basis for developing a structure of their own degree program consistent with their needs. Universities currently offering such programs may also utilize the thematic structure to reevaluate their programs and make changes, if necessary.
\end{abstract}

Keywords: Healthcare Information System, Education

\section{INTRODUCTION}

The dynamic nature of information systems necessitates constant upgrades in courses and curriculum presented by information systems departments. With rapid developments of hardware and software, better user interfaces and usability, domain specific information systems are also growing. Most notable amongst these is the field of Healthcare Information Systems. Depending on its orientation, this field is also known as Medical Information Systems, Medical Informatics, and Healthcare Information Technology (HIT). It is estimated that the market for Healthcare Information Systems (HIS) will be growing at a $20 \%$ rate in the near future (Agosta, 2009). As new fields like HIS emerge, information systems departments are faced with the challenge of developing curriculum that reflects these changes. Many universities have responded to this challenge by developing health care information systems degree programs. This paper provides a thematic structure for health care information systems programs. The structure has been developed by analyzing the coursework of current degree programs. Other issues such as the location and level of these programs have also been considered. It is hoped that such an analysis will provide universities contemplating such degree programs a structure from which they can work from. Departments currently having such programs can also use this analysis to review and make changes, if necessary to these programs.

\section{WHAT IS HEALTH CARE INFORMATION SYSTEMS?}

Healthcare Information Systems can be defined as the discipline involved with the study of applications of computer and information technologies to health care. It represents an intersection of information systems and/or computer science with the needs of the medical profession. In Figure 1, three components of HIS have been used. These are computer science knowledge, medical knowledge and business knowledge. The computer science knowledge represented in this diagram includes both theoretical and applied computer science. Business knowledge has been added to the diagram because the health care delivery systems are run as businesses and that component cannot be ignored. Depending on how the HIS program is structured, it can be represented in one of the following intersection areas:

- Computer Science Knowledge-Medical Knowledge

- Computer Science Knowledge-Medical Knowledge-Business Knowledge

The other common areas between these knowledge components are traditionally represented by other fields. The intersection between computer science knowledge and business knowledge is represented by information systems (or management information systems) while the area between business knowledge and medical knowledge is represented by fields such as medical insurance. A degree program in HIS can position itself on any of the two intersection areas identified above as HIS. Moreover, depending on the needs of the market, the target audience and the faculty background, the mix of courses in the degree program may change. For example, if the first component is chosen, some of the programs may be heavily tilted towards theoretical computer science whilst others may be tilted towards medicine. Many Bioinformatics degree programs are more computer science oriented, while many degree programs for managing health care information systems are 
oriented towards medicine. Today, Bioinformatics programs not only use computer science and molecular biology knowledge, bioinformatics research requires knowledge of mathematics, statistics, physics, chemistry, pharmacology and medicine (Ranganathan, 2005). Therefore HIS represents a wide spectrum of coursework and universities should decide where in that spectrum they would like their degree program to be located. The department of Biomedical Informatics at Vanderbilt University allows its M.S. and Ph.D. students to specialize in the following concentration areas(http://dbmi.mc.vanderbilt.edu/education/curricu lum.html, 2009), which is an excellent representation of the various areas of HIS.

- Clinical Systems - Clinical information systems are designed for the collection, storage, and manipulation of clinical information for the entire health care delivery process. This may include medical systems, pharmaceutical systems etc. Examples of such systems are electronic medical record systems for patients. Storage of patient data, patient billing etc. fall under this category (Biohealthmatics.com, 2006).

- Decision-Support Systems \& Healthcare Decision Sciences - Decision support systems are used primarily to generate case specific advice from at least two items of patient data (Wyatt and Speigelhalter, 1991). In many cases, these are based on various decision sciences models. Expert and
Artificial Intelligence (AI) based systems are included in this category. There is a long history of the development of AI based systems in medicine that dates back to the early 1970s. These may also include assistance for evidence based practice, in which knowledge is discovered and applied.

- Health Policy Management and Administration - This area focuses on information systems applications for the design, implementation and management of healthcare policies. In many cases concepts from Biostatistics and Epidemiology are used. Integration of geographical information systems (GIS) for policy development and management are also used.

- Bioinformatics for Molecular Medicine This includes the design and development of new algorithms and/or software for biomedical research. Clinical bioinformatics may be considered an extension of this area, in which molecular biology research is used for diagnosis, prevention, and treatment of diseases.

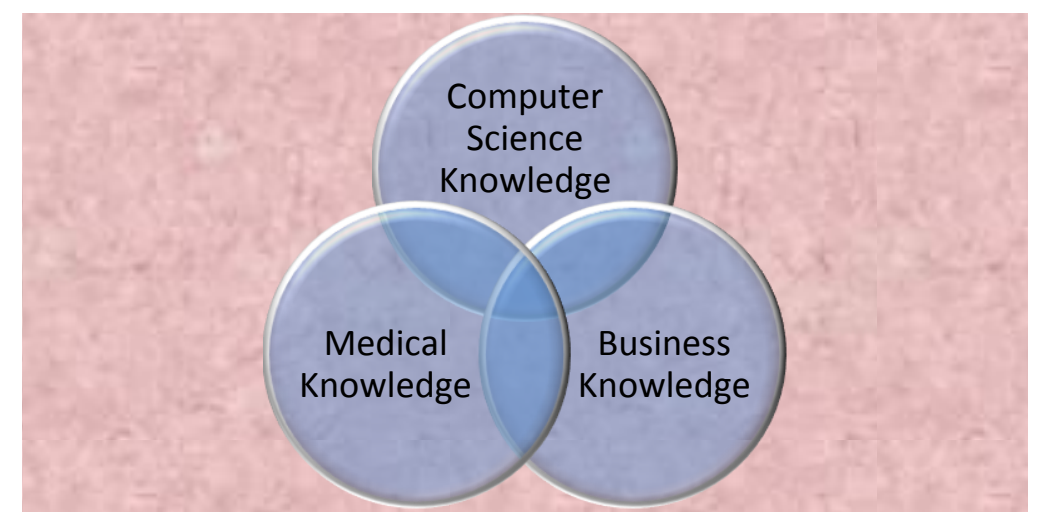

Figure 1: Components of Healthcare Information Systems Knowledge

\section{ANALYSIS OF CURRENT PROGRAMS}

Volume XI, No. 2, 2010
Based on an Internet search we have identified and located HIS programs after key word searches like 
Healthcare Information Systems, Medical Informatics etc. Based on that, we looked at three issues related to these programs; the location, level and structure of the program.

\section{Program Location}

The program location identifies the college or department that houses the program. The location of the program becomes important because that will determine the target employment market for the students and the course content. The analysis of the programs shows an interesting diversity in HIS. Of the 34 universities for which data has been collected, 22 programs are offered by a single department and the remaining 12 have joint degree programs with other departments. Of the programs offered by a single department or college, most are housed in a separate school known as Biomedical and Health Information Sciences or something similar. The next most popular location for these programs is in the colleges or schools of medicine. Clearly, independent programs in health care informatics have a very strong medical domain orientation. In independent programs, colleges of businesses and information systems departments are notably absent. In the case of joint degree programs at least one school is the medical school. This is not surprising because of the domain knowledge needed to develop HIS programs. Schools that develop joint programs

most project management courses referred to healthcare information systems projects. Once the common courses were identified, they were grouped based on themes. Two courses were considered to be in the same theme if they referred to common goals. For example, all general programming and database courses were part of information technology/information systems courses. Based on our analysis, we identified the following themes. The themes and the courses making up the themes are shown in Figure 2.

\section{Theme 1: Business/Decision Making}

Courses in this theme cover the business aspects of healthcare. It includes various aspects of project management, like initiation and planning of healthcare projects, quality, risk and project management and project closing. Even though with medical schools varied from information systems, computer science, engineering and business.

\section{Level of the Program}

Only 3 of the 34 schools for which data was collected offered an undergraduate program in health care informatics. 31 schools offered programs at the Master's level and 16 schools offered Ph.D. programs. Clearly, health care informatics is perceived mostly as a graduate degree. It has been noted though that percolation of bioinformatics curriculum down to the undergraduate level, particularly for life sciences major will allow the students to develop the multidisciplinary skills needed for their success (Ranganathan 2005).

\section{Structure of the Coursework}

In order to analyze the structure of the coursework, we looked into the course requirements of the M.S. degree programs in healthcare information systems. The M.S. degree was selected because it was the most common degree offered. The course names were used to determine whether two courses in two different programs were essentially the same. For example, Project Management and Information Technology Project Management were considered to be the same if the course coverage for Project management was primarily for information technology/information systems. In this case, though vendor management is typically considered a part of project management, some schools have opted to offer vendor management as a separate course. Workflow design issues may also be covered under this theme. In a healthcare environment, workflow design typically means how multiple transactional elements fit together (LeMoine, 2003). Such workflow analysis may be for general or for specific tasks. For example, workflow analysis may refer to how emergency room patients are admitted and treated, which may be very different from how regular patients are admitted and treated. Workflow analysis should also be integrated with systems analysis and design, which is part of the information systems/information technology themes. Many organizational issues, particularly those related to the organizational structure of hospitals and communication between the various entities that 
comprise of the organizational structure can be discussed. For example, many hospitals have a board of trustees, a director, chief medical officer etc. The reporting structure, communications and other relevant organizational issues may be covered within the context of these courses. In some cases, an organizational issues course may be integrated with other management issues related to healthcare, like issues related to leadership etc. Content covered in this section also includes other business aspects of health care, like marketing, financing and accounting. Understanding American healthcare system from a business perspective is a crucial element in such courses. For example, the relationship of insurance companies to patients and how it affects treatment decisions etc. are important components of these courses. While the legal aspects of health care may cover a wide range of topics, coverage of compliance with specific laws are extremely important. Examples of such laws are the Health Insurance Portability and Accountability Act of 1996 (HIPAA) privacy and security rules. Other important laws are the Patient Safety and Quality Improvement Act of 2005 (Kohn, L.T., et. al 2006). Ethical aspects covered in such courses deal with privacy and confidentiality as well as various established value conflicts like effectiveness versus confidentiality, and efficiency versus beneficence. Effectiveness may require medical professionals to share medical information with others (for example family members) particularly in an emergency situation while confidentiality issues prevent the sharing of such data. From a medical perspective beneficence may indicate giving the best possible care to an individual while efficiency dictates aggregation of health care resources. An example might be the use of genetically tailored medication that may be more effective and significantly reduce side effects but significantly more expensive (http://ec.europa.eu/ european_group_ethics/docs/avis13_en.pdf).

\section{Theme2: Information Systems/Technology}

The second theme in the structure for coursework covers the information systems/information technology courses. For programs seeking greater theoretical orientation, these courses may incorporate the theoretical principles of computer science to a greater degree. Systems analysis and design courses

Volume XI, No. 2, 2010 may incorporate the latest concepts, tools and techniques in this area; including object-oriented modeling and design, agile methodologies etc. In many cases, this also incorporates standards specific to healthcare information systems like the Health Level 7 (HL7). HL7 is an international standard that allows exchange, management, and integration of healthcare information (www.hl7standards.com). Apart from systems analysis and design, courses in this theme typically include both data management and database management. While data management ensures quality of the data and compliance with standards like HIPAA, database management focuses primarily on the technical aspects of managing a database; for example, use of the structured query language (SQL), integration with XML etc. Some areas of overlap are inevitable. For example, data modeling can be used in both data management as well as database management. Not only do health care professionals need the correct data, in many cases time is a crucial element in health care. Information systems developed for health care require excellent user interfaces that are consistent with the needs of the health care professional. It has been pointed out that many health care ICT systems fail because it fails to take into account user needs (Sanderson 2007). Designing user interface systems with based on sound cognitive engineering principles therefore becomes critical. As more and more healthcare information goes on-line, protecting such information becomes crucial. Students in HIS therefore should have in-depth knowledge of I.T. security. A widely used model for information systems security is the CIA triangle which sets the three primary goals for information systems security (Whitman and Mattord, 2011). These are; confidentiality, integrity and availability. A key aspect of patient confidentiality is patient identity management. This cuts across all aspects of the CIA triangle. For example, if the patient is not properly identified, it may lead to serious personal harm to the patient and significant cost to the service provider. Students should be taught about various requirements like unique individual identifiers and business processes that ensure data accuracy. Various standards like the patient identity standards have been proposed and should be taught. Incident management and auditing also becomes important issues (HIMSS, 2009). Students should realize there are stringent 
data privacy requirements. Integrated healthcare information systems are possible only through excellent communication systems and many courses related to data communications systems attest to that fact. Also, the care of patients involves many different individuals that need to share and manage patient data. Communications systems have been found to be useful both in inter-hospital and intrahospital scenarios (Coiera, 2006). Many courses in this theme also covered the management aspects of healthcare information systems, similar to the Management Information System (MIS) courses in a traditional information systems curriculum. Also included are Decision Support Systems (DSS) for health care that focus on evidence based decision support for physicians and other health support staff. In many cases, these courses may be integrated with the use of natural language processing or data mining. Data mining plays a significant role in health care information systems.

\section{Theme 3: Bioinformatics/Healthcare IS}

Courses in this theme deal directly with computer science and information systems applications in health care. Typically, an introductory course is offered that deals with concepts on how clinical systems function, the role of various information architectures and data stores, and how emerging technologies can be used in health care. Some courses also provide a broad sweep of bioinformatics, evidence based medicine and information security in health care. Most of the courses in this group focus on genomics/bioinformatics. Bioinformatics has been defined as "the application of information technology and computer science to molecular biology" (www.wikipedia.com). Fundamental knowledge of bioinformatics is essential for courses in this theme, however programming skills and access to a database plays a key role. Some of these courses focus on programming in languages like PERL, Python, and Ruby, while some others focus on the use of specialized bioinformatics tools (Honts, 2003). For example, the National Center for Biotechnology Information uses a unique search and retrieval system called Entrez. (www.ncbi.nlm.nih.gov/About/primer/bioinformatics .html). The advantage of using such specialized software is that is optimized for use with certain

Volume XI, No. 2, 2010 databases. Entrez is connected to the Entrez protein database and cross linked to the Entrez taxonomic database. Other common tools used in Bioinformatics include BLAST, a gene identification tool and RasMol which is molecular visualization software (rasMol.org). There may be a debate whether scripting languages like PERL should be emphasized or whether learning how to use software like Entrez should be emphasized. The answer may lie in the focus of the coursework. If the course is geared for teaching students how to develop newer Bioinformatics software then the focus should on scripting languages, otherwise the focus should be on specific software like Entrez. In some cases, separate courses may be offered, one focusing on scripting languages and the other focusing on software.

\section{Theme 4: Public Health}

The public health component covers courses such Tele Medicine. Telemedicine services are defined as "the use of interactive audio, video or other electronic media for the purpose of diagnosis, consultation, or treatment" (ehealthvirginia.org). Fundamental aspects of telemedicine are covered in the courses including the process of how telemedicine networks can be implemented, licensure, standards and security issues related to telemedicine and how effective telemedicine consultations may be done. Other courses in this theme may include public health surveillance, that cover how public health can be monitored, how to react to outbreak of diseases etc. Epidemiological concepts are part of these courses. Interesting aspects of public health surveillance include the use of GIS for public health monitoring (Cromley and McLafferty, 2002).

\section{Theme 5: Research Skills}

Courses in this theme address various issues related to research in HIS. These issues range from the various information sources in health care. Also, statistical concepts including concepts of multivariate statistics and research design are covered. Data analysis using a wide variety of statistical tools like SAS and SPSS and data analysis methodologies are part of this theme as well. Various scientific visualization methods that allow the data to be presented and seen in 2-D and 3-D assist health care professionals in research and diagnosis as well. 
These research skills are used in the research/seminars that students in most programs have to present. The research skills are typically tested through research seminars or research presentations.

\section{Theme 6: Personal Skills}

Successful professionals need a wide variety of personal skills like professionalism, communication and leadership skills. Many schools ensure that these skills are developed, while some others do not.

\section{Theme 7: Other}

The "other" category has an eclectic group of courses; each on its own could not be made part of a theme. The pedagogical courses in this group teach students the fundamental principles behind teaching and the learning environment for HIS students. Courses in mathematics cover fundamentals and advanced concepts in Mathematics.

\section{CONCLUSIONS}

This paper has explored various aspects of the structure of the HIS programs. The findings illustrate the fact that most of these programs were located in a separate school especially set up for biomedicine. The next most popular location for housing these programs is the school of medicine. Business schools, especially information systems departments have an opportunity to have joint degree programs with other schools especially medicine or nursing. Moreover, the lack of undergraduate programs in HIS indicates the presence of an opportunity, particularly for schools that are more focused on teaching. The themes presented in this paper can work as a guideline for the nature of coursework that may be included in HIS programs. Future research will focus on fine-tuning these themes by more sophisticated qualitative data analysis techniques like qualitative factor analysis. The authors are also collecting data on employer expectations to research into how close these various degree programs come towards meeting current employer needs.

\section{Figure 2: Themes of Health Care Information} Systems Courses

Theme1: Business/Decision Making

Project Management

IT Vendor Management

Workflow Design and Change Management

American Health Care System

Organizational Behavior

Organizational Issues in Health Care

Informatics

Management Issues in Health Care

Informatics

American Health Care System

Business of Health Care Informatics

Legal, Ethical, and Social Issues in Health

Care Informatics

Consumer Health Informatics

\section{Theme 2: Information Systems/Technology}

Introduction to Computing

Data Structures and Algorithms

Programming for Health Care Professionals

Health Care Data Management

Database Management

Usability of Health Care Systems /Cognitive

Engineering

Emerging Technologies for Health Care

Systems Theory

Health Information Systems Analysis and

Design

Object Oriented Systems Development

Standards in Health Care Informatics

Health Care Information Security

Management of Health Care Communication

Systems

Health Care I.T. Administration

Laboratory Health Information Management 


\begin{tabular}{|l|}
\hline \multicolumn{1}{c|}{ Theme 3: } \\
Bioinformatics/Health Care IS \\
Clinical Information Systems \\
Health Care Information \\
Systems/Health Informatics \\
Genomics \\
Bioinformatics \\
Electronic Health Records \\
Informatics in Life Sciences and \\
Chemistry \\
Biomedical Signal Processing \\
Image Processing \\
Nanotechnology in Healthcare
\end{tabular}

\section{Theme 4: Public Health}

Public Health Surveillance and Informatics Introduction to Tele health Principles and Foundations of Public Health Informatics

\section{Theme 5: Research Skills}

Information Sources in Health Care Informatics Research Design

Data Analysis and Management

Scientific Visualization

Health Informatics Capstone Project

Health Informatics Internships

Seminar/Research in Health Information Systems

Practicum

Independent Study

Thesis/Project

Readings in Health Care Informatics

Special Topics in Health Informatics

\section{Theme 6: Personal Skills}

Communication Skills

Professionalism

Technical Writing

Leadership Development in Health

Informatics

\section{Theme 7: Other}

Basic Mathematics

Mathematical and Logical Foundations of Informatics

Learning Environments in Health

Sciences 


\section{REFERENCES}

1. Agosta, L, "The Healthcare Information Technology (HIT) Market is Poised for Growth" - http://www.b-eyenetwork.com/view/11085. August 6, 2009.

2. Coiera, E., "Communication Systems in Healthcare", The Clinical Biochemist Reviews, Vol. 27, No. 2, May 2006, pp. 8998.

3. Cromley, E.K., and McLafferty, S.L, GIS and Public Health, The Guilford Press, New York, 2002.

4. Healthcare Information and Management Society (HIMSS), "Patient Identity Integrity", 2009, pp. 1-60.

5. Honts, J.E., "Evolving Strategies for the Incorporation of Bioinformatics within the Undergraduate Cell Biology Curriculum", Cell Biology Education, Winter 2003, pp. 233-247.

6. Kohn, L.T., et. al (editors), "Executive Summary", To Err is Human: Building a Safer Health System, The National Academies Press, Washington D.C., 2006, pp. 1-16.

7. LeMoine, D., "Going with the Flow: Interaction Design for Healthcare, Cooper Journal,

www.cooper.com/journal/2003/09/going with the flow interactio.html, 2003.

8. Ranganathan, S., "Bioinformatics Education-Perspectives and Challenges", PLoS Computational Biology, November; 1(6):e52; Published online 2005; doi: 10.1371/journal.pcbi.0010052.

9. Sanderson, P., "Designing and Evaluating Healthcare ICT Innovation: A Cognitive Engineering View", Information Technology in Healthcare; J.I. Westbrook et. al (Ed), IOS Press, Amsterdam, (2007), pp. 3-12.

10. Wyatt, J., and Speigelhalter, D., "Evaluating Medical Expert Systems:What to Test and How?", Medical Informatics, vol. 15, no. 3 (1991), pp. 205-218.
11. Whitman, M.E., and Mattord, H.J., Management of Information Security $\left(3^{\text {rd }}\right.$ Edition), Course Technology, Florence, KY, 2011. 